\title{
COMPUTATION OF THE UNORIENTED COBORDISM RING
}

\author{
EDGAR H. BROWN, JR. AND FRANKLIN P. PETERSON
}

\begin{abstract}
This note gives a set of generators for the unoriented cobordism ring and it gives a simplification of the algebra involved in Thom's computation of this ring.
\end{abstract}

In this note we give a simplification of the algebra involved in Thom's computation of the unoriented cobordism ring [3] and, along the way, a recursive formula for the generators of this ring given by Liulevicius [4].

Let $A$ denote the mod two Steenrod algebra, $A^{*}$ its dual and $\xi_{i} \in A^{*}$ the polynomial generators defined by Milnor [1]. In the following all homology and cohomology is with $Z_{2}$ coefficients. We identify $H_{*}(M O)$ with $H_{*}(B O)$ via the Thom isomorphism. The $A$ module structure of $H^{*}(M O)$ defines an $A^{*}$ comodule structure on $H_{*}(M O), \nabla: H_{*}(M O) \rightarrow A^{*} \otimes H_{*}(M O)$. The Whitney sum operation makes $H_{*}(M O)$ into an algebra and $\nabla$ is an algebra map. Let $T: \Pi^{k} B O_{1} \rightarrow B O_{k}$ be the classifying map of the product of the canonical line bundles. $T$ embeds $H^{*}(B O)$ in $H^{*}\left(\Pi^{\infty} B O_{1}\right)=Z_{2}\left[t_{1}, t_{2}, \ldots\right]$ as the algebra of symmetric functions. For a partition $w=\left\{i_{1}, i_{2}, \ldots, i_{k}\right\}$ let $s_{w}$ be the smallest symmetric function containing $t_{1}^{i_{1}} \cdots t_{k}^{i_{k}}$. Recall under the map $H^{*}(B O) \rightarrow H^{*}(B O) \otimes H^{*}(B O)$ induced by the Whitney sum,

$$
s_{w} \rightarrow \sum s_{w_{1}} \otimes s_{w_{2}}
$$

where the sum ranges over $w_{1} \cup w_{2}=w$. Let $\left\{x_{w}\right\} \subset H_{*}(B O)$ be the basis dual to $\left\{s_{w}\right\}$. Let $x_{i}=x_{\{i\}}$. (1.1) immediately yields: $x_{w}=x_{i_{1}} x_{i_{2}} \cdots x_{i_{k}}$ and hence $H_{*}(B O)=Z_{2}\left[x_{1}, x_{2}, \ldots\right]$. A straightforward calculation yields the following result of Switzer [2]: If $x=1+x_{1}+x_{2}+\ldots$ and $\xi=1+\xi_{1}+$ $\xi_{2}+\ldots$,

$$
\nabla x=\sum \xi^{i+1} \otimes x_{i} .
$$

We define elements $y_{i} \in H_{i}(M O)$ by induction on $i$ as follows: $y_{0}=1$, $y_{i}=x_{i}+\Sigma^{j<i} z_{i j} y_{j}$ where $z_{i j}$ is the $i-j$ component of $\left(\sum y_{2^{k}-1}\right)^{j+1}$ if $j \neq 2^{s}-$ 1 and $z_{i j}=0$ if $j=2^{s}-1$.

THEOREM (1.2).

$$
\begin{gathered}
H_{*}(M O)=Z_{2}\left[y_{1}, y_{2}, \ldots\right], \quad \nabla y_{i}=1 \otimes y_{i} \quad \text { if } i \neq 2^{j}-1, \\
\nabla y_{2^{j}-1}=\sum \xi_{j-k}^{2^{k}} \otimes y_{2^{k}-1} .
\end{gathered}
$$

Received by the editors February 27, 1975.

AMS (MOS) subject classifications (1970). Primary 57A70. 
The usual argument then gives

Corollary (1.3). The Hurewicz map $\rho: \pi_{*}(M O) \rightarrow H_{*}(M O)$ defines an isomorphism $\mathfrak{x}_{*} \approx Z_{2}\left[y_{i} \mid i \neq 2^{j}-1\right]$ where ${ }_{*}^{*}$ is the unoriented cobordism ring.

REMARK. With analogous definitions for the $y_{i},(1.2)$ holds for $H_{*}\left(M U ; Z_{p}\right)$, $A_{p}^{\prime}$, the algebra of reduced $p$ th powers, and 2 replaced by $p$, an odd prime.

REMARK. Using the techniques in [5], one may show that $y_{i}=x_{i}$ if $i$ $=t p^{j}-2, t=1,2, \ldots, p-1, y_{i} \in H_{i}\left(M O ; Z_{2}\right)$ for $p$ even and $y_{i} \in$ $H_{2 i}\left(M U ; Z_{p}\right)$ for $p$ odd.

Proof of (1.2). Let $N=H_{*}(M O) /\left\{x_{i} \mid i=2^{k}-1, k>0\right\}$ and let $p$ : $H_{*}(M O) \rightarrow N$ be the projection. $A^{*} \otimes N$ is a polynomial algebra on the generators $\xi_{i}$ g and $p\left(x_{i}\right), i \neq 2^{k}-1$, and it is an $A^{*}$ comodule under $\psi \otimes$ id: $A^{*} \otimes N \rightarrow A^{*} \otimes A^{*} \otimes N$ where $\psi$ is the comultiplication in $A^{*}$. Let $f$ $=($ id $\otimes p) \nabla: H_{*}(M O) \rightarrow A^{*} \otimes N . f$ is a ring homomorphism and an $A^{*}$ comodule map. We show by induction on $i$ that

$$
\begin{aligned}
f\left(y_{i}\right) & =1 \otimes p\left(x_{i}\right), \quad i \neq 2^{k}-1, \\
& =\xi_{k} \otimes 1, \quad i=2^{k}-1 .
\end{aligned}
$$

For $i=0(1.4)$ is true. Suppose (1.4) is true for $1,2, \ldots, i-1$.

$$
f\left(y_{i}\right)=f\left(x_{i}\right)+f\left(\sum\left(\left(\sum_{k} y_{2^{k}-1}\right)^{j+1}\right)_{i-j} y_{j}\right)
$$

where the above sum ranges over $j<i, j \neq 2^{k}-1, k \geqslant 0$.

$$
f\left(x_{i}\right)=\sum_{j=0}^{t}\left(\xi^{j+1}\right)_{i-j} \otimes p\left(x_{j}\right) .
$$

Thus by the inductive hypothesis,

$$
f\left(y_{i}\right)=1 \otimes p\left(x_{i}\right)+(\xi)_{i} \otimes 1
$$

and (1.4) holds for $i$.

$f$ maps the $y_{i}$ 's onto polynomial generators and hence is an epimorphism. $H_{*}(M O)$ and $A^{*} \otimes N$ have the same rank in each dimension and therefore $f$ is an isomorphism. (1.2) now follows from the comodule structure on $A^{*} \otimes N$.

\section{BIBLIOGRAPHY}

1. J. Milnor, The Steenrod algebra and its dual, Ann of Math. (2) 67 (1958), 150-171. MR 20 \#6092.

2. R. Switzer, Homology comodules, Invent. Math. 20 (1973), 97-102.

3. R. Thom, Quelques propriétés globales des variétés différentiables, Comment. Math. Helv. 28 (1954), 17-86. MR 15, 890.

4. A. Liulevicius, A proof of Thom's theorem, Comment. Math. Helv. 37 (1962/63), 121-131. MR 26 \#3058.

5. E. Brown, D. Davis and F. Peterson, $H_{*}(B O), H_{*}(B U)$ and some results about the Steenrod algebra (to appear).

Department of Mathematics, Brandeis University, Waltham, Massachusetts 02154

Department of Mathematics, Massachusetts institute of Technology, Cambridge, MASSACHUSETTS 02139 